Revue

Revue de l'histoire des religions

de Ihistoire des religions

\title{
Giuseppe Maria CROCE (a cura di), Vincenzo Tizzani. Effemeridi Romane. Volume Primo 1828-1860
}

Roma, Gangemi Editore, 2015

Laura Pettinaroli

\section{OpenEdition}

Journals

Édition électronique

URL : http://journals.openedition.org/rhr/8714

DOI : 10.4000/rhr.8714

ISSN : 2105-2573

Éditeur

Armand Colin

Édition imprimée

Date de publication : 1 mars 2017

Pagination : 190-193

ISBN : 978-2-200-93125-4

ISSN : 0035-1423

Référence électronique

Laura Pettinaroli, «Giuseppe Maria Croce (a cura di), Vincenzo Tizzani. Effemeridi Romane. Volume Primo 1828-1860», Revue de l'histoire des religions [En ligne], 1 | 2017, mis en ligne le 24 mars 2017, consulté le 08 janvier 2021. URL : http://journals.openedition.org/rhr/8714; DOI : https://doi.org/ERREUR PDO dans /localdata/www-bin/Core/Core/Db/Db.class.php L.34 : SQLSTATE[HY000] [2006] MySQL server has gone away

Ce document a été généré automatiquement le 8 janvier 2021

Tous droits réservés 


\section{Giuseppe Maria CROCE (a cura di), Vincenzo Tizzani. Effemeridi Romane. Volume Primo 1828-1860}

Roma, Gangemi Editore, 2015

Laura Pettinaroli

\section{RÉFÉRENCE}

Giuseppe Maria CROCE (a cura di), Vincenzo Tizzani. Effemeridi Romane. Volume Primo 1828-1860, Roma, Gangemi Editore, 2015, 25 cm, CDLXXI-864 p., $140 €$, ISBN

978-88-492-2615-7 (Istituto per la Storia del Risorgimento italiano - Biblioteca scientifica, Ser. II, Fonti, 104).

1 Avec cette nouvelle publication de sources, Mgr Croce force encore l'admiration du lecteur. Après les cinq superbes tomes de documents sur le père Cyrille Korolevskij (Cité du Vatican, 2007), l'archiviste émérite de l'Archivio Segreto Vaticano (ASV) livre avec ce premier volume des Effemeridi romane de Mgr Vincenzo Tizzani - une nouvelle approche biographique d'un ecclésiastique de la Rome pontificale à la période contemporaine. Si Tizzani peut a priori apparaître comme un personnage secondaire, la multiplicité de ses activités et la richesse de ses contacts tout au long de sa vie (1809-1892) le placent au cœur des événements cruciaux du XIX ${ }^{\mathrm{e}}$ siècle romain.

2 Mgr Tizzani n'est toutefois pas un inconnu. Son rôle dans l'édition des œuvres du poète romain Gioacchino Belli l'a fait connaître des spécialistes d'histoire littéraire. L'histoire religieuse s'est également intéressée à lui depuis les années 1980 : G.M. Croce avait déjà édité certains de ses textes (Archivum Historiae Pontificiae, 1985, 1986, 1987), Lajos Pásztor avait publié en deux tomes en 1991-1992 son histoire du concile Vatican I ( (diario ») et sa défense des écrits d'Antonio Rosmini à l'heure de la condamnation romaine avait également attiré l'attention (Maria Franca Mellano). 
3 L'ouvrage proposé ici, massif et d'une très belle qualité formelle, permet un progrès considérable dans la recherche sur ce personnage. En effet, il offre tout à la fois au lecteur l'aboutissement d'une enquête menée dans de nombreux fonds (dense biographie proposée dans l'introduction I), un programme de recherche (inventaires des fonds Tizzani dans l'introduction II) et met à disposition de la communauté scientifique une source de premier plan: les Effemeridi romane, journal tenu de 1828 à 1890.

4 La biographie de Tizzani (plus de 320 pages) est structurée autour de six chapitres chronologiques. Le chapitre 1, consacré à la jeunesse romaine de Tizzani, évoque son enfance dans une famille modeste (son grand-père était cordonnier sur le Campo de' Fiori). Rapidement orphelin de père, la protection de son oncle maternel, prêtre, lui permet de faire des études (Séminaire romain, Apollinaire, Sapienza). Il entre en 1832 dans le clergé régulier à San Pietro in Vincoli, au sein de l'ordre des chanoines réguliers du Latran, affecté par la période révolutionnaire mais qui dispose encore de plusieurs maisons en Italie et surtout rassemble plusieurs personnalités brillantes comme Paolo Delsignore, qui prend Vincenzo sous sa protection. C'est à la Sapienza, comme enseignant d'histoire ecclésiastique, que le jeune religieux s'illustre d'abord. Son érudition et sa curiosité, ouvertes sur de nombreux domaines, le conduisent alors à fréquenter plusieurs sociétés savantes. Il publie à cette époque des manuels qui connaissent une certaine diffusion (Institutiones historiae ecclesiasticae, 1837-1846). Le jeune religieux est cependant rapidement accaparé par des tâches administratives au sein de son ordre. Cette ascension, associée à une gestion approximative des affaires financières, nourrit des jalousies et explique son éviction/promotion comme évêque de Terni en 1843. Le chapitre 2 est consacré à cette période ombrienne: malgré son dynamisme au service de la population (éducation, apostolat), Mgr Tizzani entre en conflit avec les élites locales et doit démissionner dès 1847. Le chapitre 3, qui s'ouvre sur la révolution de 1848-49, montre l'évolution de celui qui est devenu archevêque titulaire de Nisibi et est nommé en 1850 chapelain majeur de l'armée pontificale. Tizzani déploie dans cette charge - qui vient d'être fondée en plein conflit avec le Royaume de Piémont-Sardaigne - un zèle notable, malgré une maladie qui le conduit de 1854 à 1865 à la perte intégrale de la vue. Le chapitre 4 est consacré au Tizzani « de Curie » : consulteur de l'Index depuis 1837 (où il intervient notamment sur des œuvres littéraires françaises), il joue un rôle notable dans plusieurs commissions spécialisées, notamment sur le dogme de l'Immaculée conception et la rédaction du fameux «Syllabus » des erreurs modernes. Il est aussi à l'origine d'initiatives comme la création en 1851 d'une commission d'archéologie sacrée et la réforme avortée du bréviaire romain en 1855-56. Cependant, au-delà du " monde vatican » qui s'étend des cardinaux aux employés de la Curie, Tizzani est également en contact avec des personnalités politiques (dynastie napoléonienne) et artistiques (Franz Liszt, Belli...), d'autant qu'il reste toujours actif sur le plan intellectuel, en reprenant son enseignement à la Sapienza en 1853, en participant à des sociétés savantes et en s'intéressant aux antiquités chrétiennes. Le chapitre 5 prend à bien des égards la suite du précédent, tout en se concentrant sur la période 1869-1878 "entre un concile et un conclave». Mgr Tizzani poursuit en effet son œuvre scientifique avec deux ouvrages sur le thème conciliaire (Les conciles généraux, 1867-1869 et I concili lateranesi (sic), 1878), ainsi que ses missions en Curie (commissions préparatoires au concile, tentative de médiation sur l'infaillibilité en 1870). Cependant, il doit - sur l'injonction faite par Pie IX aux professeurs de la Faculté de théologie en 1871 - quitter la Sapienza suite à l'affaire 
Döllinger. Il se consacre alors, toujours en parallèle de son action pastorale auprès de religieuses et au chapitre du Latran, à l'écriture (ou plutôt à la dictée). Le chapitre 6 embrasse la fin de sa vie sous Léon XIII : Tizzani est un témoin privilégié des évolutions de la ville de Rome. S'il s'enthousiasme pour les encouragements donnés à la recherche historique par le pape Pecci, il nourrit une certaine hostilité tant à l'égard de la vie politique italienne que de la Curie du nouveau pape, même s'il est nommé patriarche titulaire latin d'Antioche en 1886, six ans avant sa mort.

$5 \quad$ L'introduction II concerne plus directement les Effemeridi et comprend trois chapitres. Le premier offre un inventaire des principales archives Tizzani, notamment du fonds de S. Pietro in Vincoli (ASPV), où se trouvent les Effemeridi romane, mais aussi des ensembles conservés à l'ASV, à la Bibliothèque Casanatense, à Sainte-Agnès-hors-lesMurs et aux archives capitulaires de Saint-Jean-du-Latran (où est conservé un fonds inexploité de 6000 fascicules sur son activité de chapelain militaire). Le deuxième chapitre («Un mondo in un diario: Tizzani, Roma e la sua Chiesa ») est sans doute l'essai le plus significatif de l'ouvrage. On ne saurait que conseiller au lecteur d'entrer dans l'ouvrage par ce texte synthétique et enlevé où l'auteur ne cache pas sa fascination et sa tendresse pour son héros, le "cieco veggente", dont il brosse avec brio les paradoxes. La spiritualité rigoriste de Tizzani ne l'empêche pas d'être sensible aux phénomènes mystiques féminins qui se manifestent dans l'État pontifical agonisant, le prêtre érudit hostile aux Lumières n'hésite pas à défendre les penseurs considérés comme libéraux... C'est en définitive la liberté du personnage qui frappe, autant que sa perspicacité à l'égard des défis nouveaux qui s'imposent à l'Église, expliquant sans doute son évolution à rebours du courant dominant, qui le conduit à s'éloigner des intransigeants dans la seconde moitié des années 1860. Enfin le troisième chapitre présente les critères d'édition des neuf volumes manuscrits des Effemeridi.

6 L'édition des Effemeridi occupe ainsi la plus grande partie de l'ouvrage (770 pages) et est accompagnée d'un double système de notes et d'un copieux index des noms de personnes (p. 775-864). Ce volume I ne propose cependant que l'édition de la période 1828 à 1860, qui correspond à la lente mise en route de ce journal, de fait assez lacunaire jusqu'en 1856 (seules les années 1828, 1831 et 1832 sont relativement complètes). Rédigé le soir avant le coucher, dans une perspective surtout pratique, ce journal comprend des indications surtout factuelles, systématiques sur la météorologie, fréquentes sur les faits divers, l'évolution de la ville de Rome et les actualités de la Curie romaine. Au-delà de l'intérêt intrinsèque du texte, la difficulté d'accès au document (ASPV), sa graphie difficile et son codage partiel, justifient pleinement son édition. Après 1865 , le texte, dicté, devient à la fois plus lisible et moins concis.

7 Cet ouvrage, même dans l'attente du (des) volume(s) suivant(s), constitue déjà une véritable somme qui intéressera, au-delà des spécialistes de l'histoire du Risorgimento et de la ville de Rome, autant les historiens du religieux en général (notamment pour les liens avec l'histoire intellectuelle et culturelle), que les amateurs d'histoire de la Curie qui y trouveront matière à réflexion sur les mécanismes de protection et de promotion, le rôle de l'expertise et les styles de gouvernement des papes du xix siècle. Face à un tel modèle d'érudition, d'intelligence historique et de ténacité dans la recherche, on n'émettra qu'un regret minime, celui d'un découpage assez complexe de l'ouvrage conduisant à deux niveaux d'introduction et à un double système de pagination. 


\section{AUTEURS}

\section{LAURA PETTINAROLI}

Institut catholique de Paris. 